\title{
Application of different imaging methods for the localization of cerebrospinal fluid rhinorrhea: a comparative study
}

\author{
Xiang Zhai ${ }^{1 \#}$, Liang $\mathrm{Li}^{2 \#}$, Xing Lu ${ }^{3 \#}$, Hai-Yan $\mathrm{Li}^{3}$, Xiao-Lu Yang ${ }^{4}$, Xu-Dong Wang ${ }^{1}$ \\ ${ }^{1}$ Department of Maxillofacial \& E.N.T, Tianjin Medical University Cancer Institute and Hospital, National Clinical Research Center for Cancer, \\ Tianjin' Clinical Research Center for Cancer, Key Laboratory of Cancer Prevention and Therapy, Tianjin, China; ${ }^{2}$ Department of Otolaryngology, \\ Tianjin Children's Hospital, Tianjin University Children's Hospital, Tianjin, China; ${ }^{3}$ Department of Otolaryngology, Tianjin Huanhu Hospital, \\ Tianjin, China; ${ }^{4}$ Department of Otolaryngology, Bethune International Peace Hospital, Shijiazhuang, China \\ Contributions: (I) Conception and design: X Zhai; (II) Administrative support: XD Wang; (III) Provision of study materials or patients: X Lu; (IV) \\ Collection and assembly of data: L Li, XL Yang; (V) Data analysis and interpretation: X Lu, HY Li; (VI) Manuscript writing: All authors; (VII) Final \\ approval of manuscript: All authors. \\ \#These authors contributed to the work equally as co-first authors. \\ Correspondence to: Xu-Dong Wang. Department of Maxillofacial \& E.N.T, Tianjin Medical University Cancer Institute and Hospital, National \\ Clinical Research Center for Cancer, Tianjin' Clinical Research Center for Cancer, Key Laboratory of Cancer Prevention and Therapy, Tianjin, \\ China. Email: wxd.1133@163.com.
}

Background CSF rhinorrhea is a type of CSF leakage caused by an aseptic abnormal passage between the subarachnoid space and the adjacent sinus and nasal cavity due to a cranial dural defect. At present, the value of computed tomography cisternography (CTC) in locating CSF rhinorrhea has been widely recognized, and magnetic resonance hydrography $(\mathrm{MRH})$, as a heavy T2-weighted water imaging, plays a pivotal role in showing the location of the leak. In this paper, we retrospectively summarize the imaging manifestations seen at our hospital of patients with clinically confirmed CSF rhinorrhea at the skull base and evaluate the diagnostic value of different imaging methods in the localization of CSF rhinorrhea by means of preoperative imaging analysis using CTC and MRH.

Methods: Fifty-five patients with CSF rhinorrhea admitted to our department from October 2016 to January 2021 were retrospectively analyzed. The patients' conventional CT, CTC, and MRH imaging data were compared, and the location of the leak determined preoperatively matched the location of the leak found during surgery. Moreover, there was no recurrence during the follow-up period of 4 months to 3 years.

Results: There were statistically significant differences between the diagnostic positivity rate of CTC and spiral CT $\left(\chi^{2}=16.755, \mathrm{P}<0.00\right)$, and between the diagnostic positivity rate of cranial MRH and spiral CT $\left(\chi^{2}=6.338,6.338=0.01\right)$, and no statistically significant difference between the diagnostic positivity rate of CTC and cranial MRH $\left(\chi^{2}=2.625, \mathrm{P}=0.1\right)$.

Conclusions: The combined use of imaging techniques has important practical significance for the proper treatment and prognostic evaluation of CSF rhinorrhea. CTC has the highest positive rate for the diagnosis of CSF rhinorrhea, followed by MRH, while spiral CT is safer. CTC and MRH can promote the diagnostic rate in determining the location of CSF rhinorrhea, and selective combined application can be an important guide to surgery. Keywords: Magnetic resonance hydrography (MRH); computed tomography cisternography (CTC);
cerebrospinal fluid rhinorrhea; diagnosis

Submitted May 24, 2021. Accepted for publication Aug 11, 2021.

doi: 10.21037/apm-21-1994

View this article at: https://dx.doi.org/10.21037/apm-21-1994

(c) Annals of Palliative Medicine. All rights reserved. 


\section{Introduction}

Cerebrospinal fluid (CSF) rhinorrhea is a type of CSF leakage presenting as egress of CSF from an abnormal open communication formed by arachnoid and dura mater and defective skull sections leading into the nasal cavity and sinuses, when there is a dural and osseous defect at the anterior skull base. Most patients recover after conservative treatment, but a few patients remain incapacitated for a long time or experience repeated CSF leaks. The main manifestation of CSF rhinorrhea is a persistent and uncontrollable unilateral or bilateral watery nasal discharge aggravated by bending over or Valsalva maneuver. Common causes of CSF rhinorrhea include: trauma, medically induced rhinorrhea, skull base tumors, congenital developmental malformations, and spontaneous CSF rhinorrhea. Due to communication between the subarachnoid space and the nasal cavity, CSF rhinorrhea can lead to secondary intracranial infection, meningitis, and low intracranial pressure. The high risk of these fatal complications emphasizes the importance of early detection, accurate diagnosis, and timely repair of CSF leaks, especially for patients with CSF rhinorrhea who have failed conservative treatment and require active surgical treatment. Accurate diagnosis and precise location of the leak are key to the success of CSF rhinorrhea repair treatment. At present, the value of CT cisternography (CTC) in locating CSF rhinorrhea has been widely recognized, and magnetic resonance hydrography $(\mathrm{MRH})$, i.e., heavy T2-weighted water imaging, plays a certain role in showing the location of the leak. Imaging is useful for surgeons to plan surgical access, avoid damage to vital structures, establish graft beds and repair techniques, and avoid craniotomy as much as possible. In this article, we retrospectively summarize the imaging manifestations at our hospital of patients with clinically confirmed CSF rhinorrhea at the skull base when leak verification by surgery was performed to compare the diagnostic and treatment value of different imaging methods in guiding the location of CSF rhinorrhea. We present the following article in accordance with the STROBE reporting checklist (available at https://dx.doi. org/10.21037/apm-21-1994).

\section{Methods}

\section{General data}

We retrospectively analysed 55 patients with CSF rhinorrhea admitted to the Department of Otolaryngology,
Tianjin Huanhu Hospital, from October 2016 to January 2021. Among them, 30 cases were male and 25 cases were female, aged 8 to 73 years, with a median age of 51 years. The duration of disease ranged from 5 days to 14 years, with a median time of 3 months. There were 28 cases of spontaneous CSF rhinorrhea, 10 cases of traumatic CSF rhinorrhea, 13 cases of medically induced CSF rhinorrhea after surgery, and 4 cases of CSF rhinorrhea during drug treatment or radiotherapy for pituitary tumors. There were 8 cases with preoperative signs of meningitis such as fever. The inclusion criteria were patients with intermittent or persistent nasal leakage and a glucose content of $\geq 1.66 \mathrm{mmol} / \mathrm{L}$ in the CSF confirmed using biochemical examination. The study was approved by the Ethics Committee of Clinical Investigation in the Tianjin Huanhu Hospital (No. 2021-049: the registration number of ethics board). All procedures performed in this study involving human participants were in accordance with the Declaration of Helsinki (as revised in 2013). Individual consent for this retrospective analysis was waived.

\section{Research methods}

\section{CTC}

The patient was placed in the lateral position, and a routine lumbar puncture was performed with a No. 9 puncture needle. The outflow of CSF from the puncture needle indicated that the puncture needle entered the spinal canal, $10 \mathrm{~mL}$ of CSF was released, and then $10-15 \mathrm{~mL}$ of iohexol injection was injected into the lumbar cistern. Then the patient was placed in the knee-chest position with the head tilted forward and the forehead touching the examination bed, such that the anterior skull base was located in the lowest position of the subarachnoid space; also the hips were elevated, in order to note whether there was CSF leakage. After 15-20 minutes, the patient was moved to the CT examination room in this position for a coronal thin layer scan of the anterior skull base with a Philips Brilliant (Amsterdam, The Netherlands) 256-layer spiral CT machine. The scan parameters were $120 \mathrm{kV}, 200 \mathrm{~mA}$, bulb rotation speed $0.27 \mathrm{~s} / \mathrm{r}$, layer thickness $2.4 \mathrm{~mm}$, and spacing $2.4 \mathrm{~mm}$. After scanning, axial, coronal, and sagittal reconstructions were performed.

\section{Cranial MRH}

The examination was performed with a Siemens (Munich, Germany) Avanto 1.5 T superconducting magnetic resonance scanner and an eight-channel phased-array 
cranial coil. Patients were instructed to assume a supine position, with some in a lateral or prone position, with head first access. The subject was first subjected to conventional transaxial MRI, including spin-echo imaging (SE) T1-weighted image (T1WI), turbo spin-echo imaging (TSE) T1-weighted image (T2WI), and fat-suppressed TSE T2WI sequences (conventional scan parameters), followed by constructive interference in steady state (CISS) sequences (repetition time, TR $8.56 \mathrm{~ms}$, echo time, TE $3.91 \mathrm{~ms}$, layer thickness $0.7 \mathrm{~mm}$, FOV $180 \times 180)$, and then followed by a T2WI 3DSPACE sequence (TR 3,200 ms, TE 386 ms, layer thickness 1.0 mm, FOV 250×250). Finally, the acquired three-dimensional (3D) volume images were reconstructed in maximum intensity projection at a syngo via a processing workstation.

\section{Leakage determination}

The otorhinolaryngologist observed the images, evaluated the fluid accumulation in the sinus cavity of the nasal sinus, determined whether there was CSF rhinorrhea and the location of the leak, analyzed and recorded the specific location of the nasal leak, and observed whether there were other abnormalities within the scanning range. If there were CSF rhinorrhea, bone discontinuity of the sinus wall, and local soft tissue changes, and contrast agent deposition in the sinus cavity could be observed during the CTC examination, meanwhile, loss of continuity of the dural or skull at the base of the skull and meningoencephalocele at the defect could be seen in cranial MRH examination. If there was a linear high-signal shadow between the intracranial high-signal CSF and the high-signal fluid shadow in the nasal cavity or sinus, the connection was considered to be the location of the leak.

\section{Nasal endoscopic repair surgery}

(I) In patients with spontaneous or traumatic CSF rhinorrhea after conservative treatment failed, the first choice was to repair the leak via nasal endoscopy. This entailed epidural repair if the leak was located in the frontal sinus, and subdural repair if the nasal leak was combined with intracranial lesions. The leak was explored according to preoperative imaging data, the number and location of the leaks were determined under direct vision, the mucosa and bone around the leaks were removed, the herniated brain tissue was cauterized, and then the leaks were repaired in a multilayer repair.

(II) In patients with medically induced CSF rhinorrhea, all patients with pituitary adenoma were reviewed for head MRI within 1 week and 3 months after surgery, and once a year thereafter. Cranial MRH was reviewed immediately when CSF rhinorrhea occurred during follow-up, while routine blood tests, lumbar puncture CSF examination, CTC, and nasal endoscopy were also performed.

The surgical approach was selected according to the preoperative localization of the leak. The bones around the leak and its surrounding skull base were exposed, and damage to the sinus was minimized as much as possible. All patients were treated in the prone position under general anesthesia, and the appropriate surgical approach was selected according to the preoperative location of the leak. If the leak was suspected to be located in the ethmoidal roof, the ethmoid sinus was opened to expose the anterior skull base bone and the leak. If the leak was located in the cribriform plate, the middle turbinate was removed outward with a detacher to fully expose the leak. If the leak was located in the sphenoid sinus or the floor of the sella turcica, the middle turbinate was used as a marker to locate the opening of the sphenoid sinus and enter the sphenoid sinus, and a large part of the anterior wall of the sphenoid sinus was removed. Care should be taken to protect the optic nerve and internal carotid artery when dealing with the anterior wall of the sphenoid sinus. For leaks located in the lateral wall of the sphenoid sinus, a trans-pterygoid approach was needed, that is, the posterior margin of the maxillary sinus ostium was enlarged first, the posterior wall of the maxillary sinus was opened, the pterygopalatine fossa was exposed, the neurovascular in the pterygopalatine fossa was moved externally, and the root of pterygoid process to the lateral wall of the sphenoid sinus was exposed and abraded from interior to lateral and from anterior to posterior. For leaks located in the frontal sinus, a trans-frontal sinus approach was used, while attention was paid to the frontal sinus ostium and the size of the leak. In those cases where the CSF rhinorrhea around the frontal sinus could not be seen and where there were difficulties in enlarging the frontal sinus ostium, an external Lynch incision was added to assist the surgery. All repair materials were taken from autologous tissues. The repair materials for medical CSF rhinorrhea were the mucous membrane of the nasal septum with or without pedicle, and the repair materials for spontaneous and traumatic CSF rhinorrhea were the free temporalis muscle and temporalis fascia. After 
Table 1 Comparison of the positive diagnostic rates of CTC, cranial MRH, and spiral CT examinations

\begin{tabular}{lccc}
\hline Items & Positive (cases) & Negative (cases) & Positive rate (\%) \\
\hline CTC & 44 & 3 & $93.6^{*}$ \\
MRH & 33 & 7 & $82.5^{\#}$ \\
Spiral CT & 32 & 23 & 41.8 \\
\hline
\end{tabular}

${ }^{*}, \chi^{2}=16.755, \mathrm{P}<0.01 ;{ }^{\#}, \chi^{2}=6.338, \mathrm{P}=0.01$. CTC, computed tomography cisternography; MRH, magnetic resonance hydrography; CT, computed tomography.

the repair was completed, a gelatin sponge was applied to the outermost layer of the repair material to prevent the displacement of the repair material during filling. Finally, the nasal cavity was filled with iodoform gauze to fasten the repair material.

Postoperative management included semi-sitting of the patient for 4-7 d, avoidance of activities that raise intracranial pressure after surgery, low-salt diet, and maintenance of electrolyte balance. To prevent meningitis, third-generation cephalosporins were administered perioperatively until the withdrawal of the iodoform gauze $10-14$ d postoperatively. Postoperative follow-up was 6-36 months.

\section{Results}

All patients completed the examination successfully. 47 patients underwent CTC, 40 patients underwent MRH, and 55 patients underwent spiral CT. 44 leaks were detected by CTC, 33 by MRH, and 32 by high-resolution CT. The leak was located in the sphenoid sinus in 10 cases, in the ethmoid sinus in 24 cases, in the saddle base in 12 cases, and in the frontal sinus in 9 cases.

\section{Statistical analysis}

Data analysis was performed with SPSS10.0 statistical software. Measurement data using analysis of variance, intergroup comparison is the $t$ test, calculators information is chi-square test. $\mathrm{P}<0.05$ was considered as significant. There were statistically significant differences between the diagnostic positivity rate of CTC and spiral CT $\left(\chi^{2}=16.755\right.$, $\mathrm{P}<0.00)$, and between the diagnostic positivity rate of cranial MRH and spiral CT $\left(\chi^{2}=6.338,6.338=0.01\right)$, and no statistically significant difference between the diagnostic positivity rate of CTC and cranial $\mathrm{MRH}\left(\chi^{2}=2.625, \mathrm{P}=0.1\right)$, see Table 1.

All 55 patients in this group underwent nasal endoscopic repair of CSF rhinorrhea, and all patients were hospitalized for 3 to 5 weeks, with a mean of 4 weeks; postoperative follow-up ranged from 1 month to 5 years, with a mean of 3 years; 50 patients underwent 1 nasal endoscopic repair, 1 case underwent 2 nasal endoscopic repairs at 5 and 15 months after cranial trauma; 1 case underwent 2 nasal endoscopic repairs at 3 months and 3 years after pituitary adenoma resection; 1 case underwent pituitary adenoma resection and 3 nasal endoscopic repairs at 6, 32 and, 37 months after gamma knife radiotherapy; and 1 case was operated for nasal polyps 2 years ago in another hospital and was operated for rhinorrhea with Pathological findings showed low differentiated squamous cell carcinoma. Concerning the latter case, 8 months after the operation, CT showed that the tumor had invaded the adjacent bone and the right periorbital fat space, the nasal leak was detected after the intraoperative resection of the tumor, and thus 2 nasal endoscopic repairs were performed. One case with a pituitary tumor through transfrontal craniotomy, and 2 nasal endoscopic repairs were performed at 24 and 35 months after gamma knife radiotherapy. There was no recurrence of CSF rhinorrhea during the follow-up period.

\section{Typical case report}

\section{Case 1}

The patient, a 58-year-old female, was admitted to our hospital on August 17, 2020, mainly due to intermittent flow of clear watery material from the left nose for six months, without nasal congestion, runny nose, irritating sneezing, headache, fever, nausea, vomiting, diplopia, or visual impairment. Previous history of hypertension and diabetes last for 6 months. Physical examination after admission: clear consciousness, fluent speech, no abnormalities in cardiopulmonary and abdominal examination, a small amount of secretion visible in the left nasal cavity, the bilateral middle nasal meatus were unimpeded, mucosa was smooth in the nasopharynx, and 

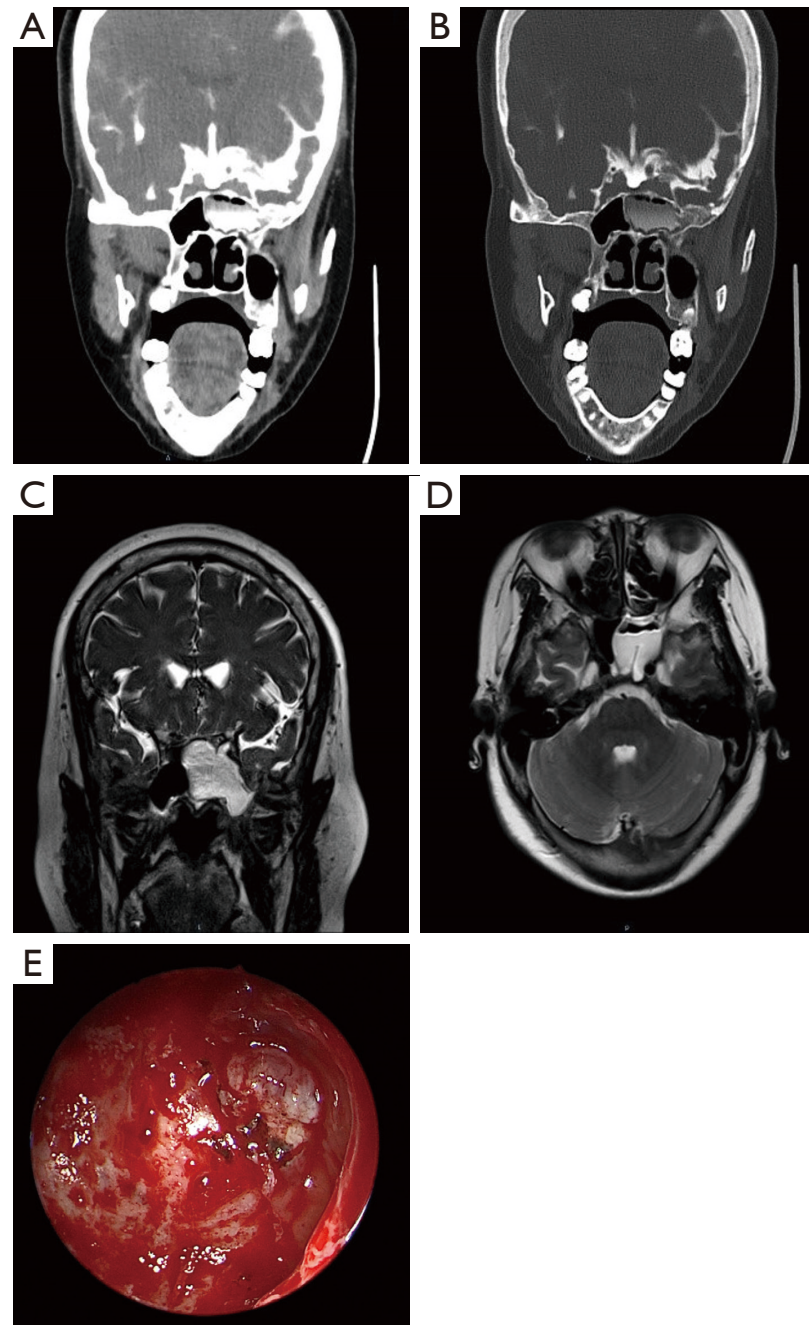

Figure 158 -year-old woman with idiopathic intermittent watery nasal discharge on left side. (A,B) Computed tomography cisternography: bone defect in the sinus wall of the left sphenoid sinus with contrast into the left sphenoid sinus; (C,D) cranial magnetic resonance hydrography: liquid signal in the left ethmoid sinus and left sphenoid sinus, with the left sphenoid sinus obvious; (E) meningoencephalocele in the lateral wall of the left sphenoid sinus with pulsatile flow of clear fluid is revealed using $70^{\circ}$ microscopy reveals.

no neoplastic organisms were seen. CTC: bone defect in the sinus wall of the left sphenoid sinus and contrast agent entered the left sphenoid sinus (Figure 1A,1B). Cranial MRH: T2WI showed liquid signal in the left ethmoid sinus and the left sphenoid sinus, and the left sphenoid sinus was prominent. The left olfactory bulb was narrowed, and a linear high signal was visible below, as was also the case for the left ethmoid sinus. The axial view showed full elevation in the left pharyngeal recess (Figure 1C,1D). CSF routine: Pandy's test negative, light yellow, slightly turbid, total cells $4 \times 10^{6} / \mathrm{L}$, leukocytes $4 \times 10^{6} / \mathrm{L}$. CSF biochemistry: glucose $4.04 \mathrm{mmol} / \mathrm{L}$, protein $0.49 \mathrm{~g} / \mathrm{L}$, lactate $1.6 \mathrm{mmol} / \mathrm{L}$, chloride $129 \mathrm{mmol} / \mathrm{L}$. Endoscopic CSF rhinorrhea repair, sphenoid sinus opening, ethmoid sinus opening, and fascia lata removal were performed on August 20, 2020. During the operation, the nasal endoscopy revealed a deviated nasal septum, unobstructed nasal meatus bilaterally, and clear fluid flow from the left sphenoethmoidal recess. Part of the left middle turbinate was resected; the anterior and posterior groups of the ethmoid sinuses and maxillary sinuses were opened; the sphenopalatine artery was electrocoagulated; the lamina perpendicular ossis palatina and the pterygoid process were partly abraded; and the corresponding branches of the sphenopalatine ganglion, pterygoid nerve, and maxillary artery were dissected and separated to retain the pterygoid nerve. The anterior wall of the sphenoid sinus was enlarged laterally, a foramen rotundum was seen, and the meningoencephalocele with pulsatile flow of clear fluid was seen in the lateral recess of the left sphenoid sinus (Figure 1E). The encephalomeningocele and their surrounding mucosa were electrocoagulated to fully expose the bone window, and roundish defects with a diameter of about $5 \mathrm{~mm}$ were seen. The edge of the defect was scratched to prepare a transplant bed, the prepared muscle was inserted into the defect to repair it, and the fascia lata was applied externally. The exposed pterygoid nerve and the repaired fascia were covered with artificial meninges, and no CSF flow was observed. The patient was hospitalized for $19 \mathrm{~d}$ and was considered cured at discharge. The patient is currently being followed up for 8 months, and no recurrence of CSF leakage has been observed thus far.

\section{Case 2}

The patient, a 68 -year-old female, was admitted to our hospital on December 18, 2020, mainly because of a clear watery discharge from the right nasal cavity for 1 week, without nasal congestion, runny nose, irritating sneezing, headache, fever, nausea, vomiting, diplopia, or visual impairment. There has been previous history of hypertension for 20 years. After admission, a physical examination showed: clear consciousness, fluent speech, no abnormalities in cardiopulmonary and abdominal examination, a small amount off clear discharge from the right nasal cavity, the bilateral middle nasal meatus were clear, presence of smooth mucosa in the nasopharynx, and 

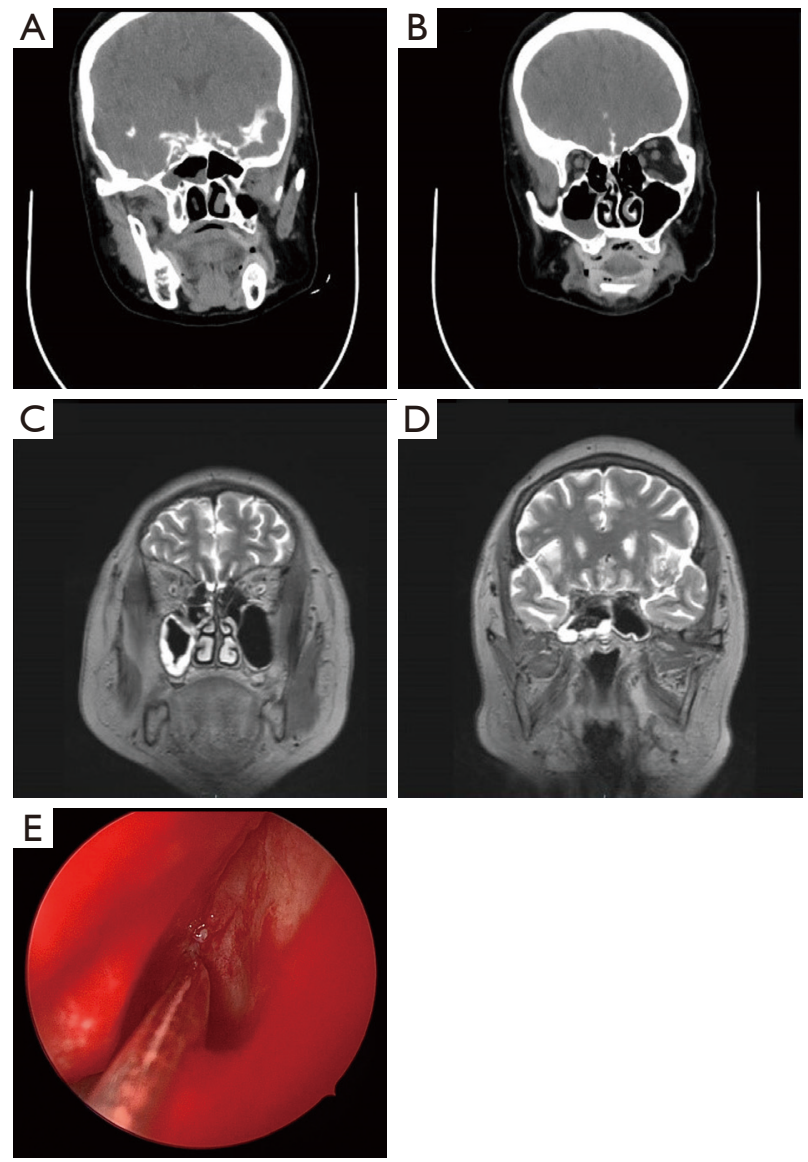

Figure 2 68-year-old woman with idiopathic watery nasal discharge on right side. $(\mathrm{A}, \mathrm{B})$ computed tomography cisternography: cloudy right frontal sinus, sphenoid sinus and bilateral maxillary sinus suggesting inflammation; soft tissue shadow with contrast diffusion in right ethmoid sinus suggesting inflammation with cerebrospinal fluid (CSF) rhinorrhea, bone structure of sinus wall of right sphenoid sinus was discontinuous; (C,D) cranial magnetic resonance hydrography: small amount of localized CSF accumulation in the anterior part of the base of the anterior cranial fossa, long T2-signal in the right maxillary sinus, right ethmoid sinus, and right sphenoid sinus; (E) intraoperatively, the mucosa of the right nasal top cribriform plate near the crista galli was seen to be slightly elevated, with pulsatile flow of clear fluid.

no neoformation were seen. CTC: cloudy right frontal sinus, sphenoid sinus, and bilateral maxillary sinus suggesting inflammation; soft tissue shadow with contrast diffusion in the right ethmoid sinus suggesting inflammation with CSF rhinorrhea; and bone structure of the right sphenoid sinus wall was discontinuous (Figure 2A,2B). MRH: small amount of localized CSF collected at the anterior part of the base of the anterior cranial fossa; and long T2-signal in the right maxillary sinus, right ethmoid sinus, and right sphenoid sinus (Figure 2C,2D). CSF routine: Pandy's test negative, colorless and clear, leukocytes $10 \times 10^{6} / \mathrm{L}$. CSF biochemistry: glucose $3.01 \mathrm{mmol} / \mathrm{L}$, protein $0.41 \mathrm{~g} / \mathrm{L}$, lactate $1.6 \mathrm{mmol} / \mathrm{L}$, chloride $130 \mathrm{mmol} / \mathrm{L}$. Endoscopic CSF rhinorrhea repair and ethmoid sinus opening were performed on December 24, 2020. Intraoperatively, nasal endoscopy revealed a deviated nasal septum with unobstructed nasal meatus on each side and clear fluid flow from the right olfactory cleft. The right middle turbinate was displaced to the lateral side, and the mucosa of the right cribriform plate near crista galli was seen to be slightly extruded, with pulsatile flow of clear fluid. The mucosa around it and the adjacent ethmoid sinus were excised, and a bony defect of the cribriform plate was seen, the dura mater was exposed, and a round dural defect was noted. The edge of the defect was scratched to make a transplant bed, the defect was repaired with a muscle plug, 2 layers of fascia were applied outside of it, and no CSF flow was seen (Figure 2E). The patient was hospitalized for $21 \mathrm{~d}$ and was considered cured at discharge. Postoperative head CT: mixed density shadow in bilateral sphenoid sinuses and right ethmoid sinus, seen after CSF rhinorrhea repair. The patient is currently being followed up for 3 months, and no recurrence of CSF leak has been observed.

\section{Discussion}

CSF rhinorrhea is a type of CSF leakage caused by an aseptic abnormal passage between the subarachnoid space and the adjacent sinus and nasal cavity due to a cranial dural defect, the main symptom of which is persistent and uncontrollable unilateral or bilateral watery nasal discharge aggravated by bending over or Valsalva maneuver (1). Due to the spread of infection from the nasal cavity, these patients are at risk of developing meningitis, with infection rates ranging from $19-50 \%$ in patients with persistent rhinorrhea (2). Using antibiotic therapy, bacterial meningitis has a mortality rate of up to $33 \%$ in adults and can be severe enough to cause seizures, encephalopathy, or cranial nerve damage (3). The high risk of these fatal complications emphasizes the importance of early detection, accurate diagnosis, and timely repair of CSF leaks. Endoscopic surgery for CSF leaks has a high success rate and a significantly lower incidence of complications such as wound infection, sepsis, and meningitis compared with open CSF leak repair, making it currently the first choice for the treatment of CSF leaks. Thorough imaging prior to 
endoscopic repair is necessary to assess the anatomy of the area and to determine the precise location of the leak and the size of the bone defect. This examination facilitates the surgeon's planning of the surgical approach to avoid damage to vital structures, establish the transplant bed and repair technique, and avoid craniotomy as much as possible.

The anterior cranial fossa accommodates the anterior inferior portion of the frontal lobe and lies above the ethmoid sinus, frontal sinus, and orbit. The thin walls of the anterior ethmoid artery entry site, ethmoid sinus, and sphenoid parietal wall are vulnerable to injury and erosion, which may lead to CSF rhinorrhea (4). The cribriform plate is the best site of occurrence. Unilateral lesions of the olfactory fossa are rare; if present and the sinus structures are clear, and without history of surgery or inflammation associated with it, the site of CSF rhinorrhea should be suspected. Sphenoid sinus pneumatization may extend anteriorly into the anterior clinoid process, laterally into the greater wing of sphenoid bone, downward into the pterygoid process, and posteriorly into the slope. In the case of lateral hyperpneumatization of the sphenoid sinus, the area of the sphenoid sinus beyond the vidian rotundum (VR) line, which connects the pterygoid canal to the foramen rotundum, is a common area of spontaneous CSF rhinorrhea (5). Hyperpneumatization of the sphenoid sinus leads to bone thinning and adjacent arachnoid granules associated with CSF rhinorrhea.

Ommaya et al. (6) classified traumatic CSF rhinorrhea according to presence or absence of the following: trauma as a result of accidental and medically induced injuries, and non-traumatic as occurring from congenital defects, potential tumors, or spontaneous CSF rhinorrhea, with the latter having a high recurrence rate of surgical repair due to various factors such as elevated intracranial pressure, multiple skull base defects, and meningoencephalocele. We focus on the relevant anatomical sites and highlight both the clinical features and imaging manifestations of traumatic and non-traumatic CSF rhinorrhea; findings may influence the surgical outcome.

Traumatic CSF rhinorrhea is due to the thin bone of the posterior wall of the frontal sinus and cribriform plate being in close proximity to the dura mater, and the simultaneous rupture of the bone lamella and dura mater in skull base fractures (7), which account for approximately 80-90\% of CSF leaks, and approximately $10 \%$ to $30 \%$ of skull base fractures combined with CSF leakage (8). Fractures involving the anterior skull base, i.e., the cribriform plate, ethmoidal roof, and posterior wall of the frontal bone, are usually associated with CSF leakage and central skull base fracture involving the sphenoid wall. Extension into the anterior cranial fossa, in particular, may be due to the tight attachment of the intrinsically thin cribriform plate and the dura mater in the ethmoidal roof region. Fractures of the frontal sinus through the posterior wall of the frontal sinus and fractures of the central skull base through the sphenoid sinus may also result in CSF rhinorrhea. Imaging manifestations include comminuted fractures without displacement or penetrating wound to the skull base, Moreover, the pneumocephalus implies skull base fracture and dural defect. Schlosser's study shows that eighty percent of patients present with CSF rhinorrhea in the first 48 hours and $95 \%$ in the first 3 months after trauma (9). The diagnosis of the initial onset of CSF rhinorrhea may be delayed due to the initial bleeding that seals the defect and can increase when the patient recovers and engages in more activities. However, a small percentage of patients present with CSF rhinorrhea months or years after trauma, triggered by atrophy of granulation tissue or because of progressive erosion of the dura mater by bone fragments. High-resolution computed tomography imaging alone has been shown to be effective in cases of post-traumatic leakage with an apparent single fracture. However, for patients with delayed presentation, complete imaging evaluation may be necessary after hematoma clearance, wound contraction, or osteonecrosis. Ten of our cases with CSF rhinorrhea were due to traumatic brain injury, and all presented with delayed CSF rhinorrhea occurring 1 month to 3 years after trauma.

Iatrogenic derived CSF rhinorrhea can occur secondary to skull base or transnasal endoscopic surgery (10). In addition to the sella turcica and parasellar, the endonasal transsphenoidal approach for skull base lesions has been significantly expanded to the scope include the clivus, anterior skull base, craniovertebral junction, and anterior border of foramen magnum, with CSF rhinorrhea as a potential complication for endoscopic treatment of these lesions. It should be emphasized that it is necessary to observe the pneumatization of the clivus, dorsum sellae, and planum sphenoidale during the operation to avoid the possibility of iatrogenic CSF rhinorrhea during transsphenoidal surgery. Most iatrogenic leaks occur in the first 2 weeks postoperatively and require lumbar cistern drainage to reduce intracranial pressure. If repair is indeed necessary, only CT imaging is usually required preoperatively, as both clinical presentation and imaging can suggest a leak associated with the surgical site. The application of CTC during the postoperative period may 
be risky due to the presence of stuff e and hematoma in the operative area. In the preoperative evaluation of suprasellar tumors and paraclinoid aneurysms, special attention needs to be paid to the sinus pneumatization and its extension into the anterior clinoid process. Potential sites of injury include the olfactory fossa at the perpendicular plate of the middle turbinate and the ethmoid roof during episodes of chronic sinusitis. Other causes include secondary alterations or infections from previous craniotomy sinus stuff such as biogels and CSF rhinorrhea due to inadequate sinus drainage. The incidence of CSF rhinorrhea after sinus surgery has been reported to be only $0.5 \%$ (10). In our group, there was 1 case of CSF rhinorrhea caused by postoperative sinusitis and 8 cases of CSF rhinorrhea caused by postoperative pituitary tumor, the latter of which was considered to be related to unfirm reconstructed stuff of the wall of the sphenoid sinus at the base of the sella turcica during the endonasal transsphenoidal approach to pituitary tumor surgery, infection of the stuffing, and tumor recurrence factors.

The least common category of CSF rhinorrhea is caused by tumor invasion of the skull base, osteonecrosis, or other erosive processes (e.g., Gorham-Stout disease) and is considered to be associated with intra-and extracranial communicating tumors and with chronic inflammatory processes in skull base bone invasion. Pituitary macroadenomas are usually prone to rhinorrhea after drug therapy or radiotherapy, due to structural and functional damage to the sinus mucosa caused by radiotherapy, or due to tumor shrinkage after drug therapy. Three cases of CSF rhinorrhea were caused by post-gamma knife radiotherapy for pituitary macroadenoma in our group and occurred initially 2-23 months after gamma knife treatment, and 1 case was treated with bromocriptine.

Spontaneous CSF rhinorrhea relates to congenital bony defects without skull base pathology , congenital malformations, or history of trauma or surgery as etiology; most of CSF leaks are caused by potential idiopathic intracranial hypertension (IIH) (11). The pathogenesis of spontaneous CSF rhinorrhea includes the following: idiopathic sustained slow increase in intracranial pressure leading to filling of the medial concave surface of the skull or sinus bone by arachnoid granules; easy resorption and destruction of the bone vulnerable places; herniation and rupture of the meninges through the bone defect resulting in CSF leak; fluctuating or sustained increase in CSF pressure and herniation of the meninges at the bone with weakness resulting in CSF leak; abdominal obesity leading to increased abdominal pressure; obstruction of intracranial venous blood return to the heart and increased cerebral blood volume, resulting in persistent benign intracranial hypertension and CSF reabsorb inhibition. Arachnoid granular filling and IIH-induced meningoencephalocele usually involves thin bones or being adjacent to hyperventilated sinuses, such as the ethmoidal roof and cribriform plate, lateral sphenoid recess, base of anterior and middle cranial fossa, such as diaphragm sella, sphenoid plateau, or ethmoid sinus. Typical MRI presentations include enlarged and tortuous optic nerve sheaths, posterior scleral flattening, and protrusion of the optic papillae into the eyeball; and herniated arachnoid granules in the middle cranial fossa leading to skull base invasion, empty sella, and meningoencephalocele. The hyperpneumatized lateral sphenoid wall is a common site for spontaneous CSF rhinorrhea combined with cranial hypertension. In the present study, meningoencephalocele could be found in 7 of 28 cases of CSF rhinorrhea.

An important part of the diagnostic process is the localization of the leak. Spiral CT can show bone defects or fractures, and the presence of fluid in the sinus cavity where these bone defects located at often indicates the location of leakage and provides information on skull base fractures, providing the surgeon with information on skeletal variations and surgical treatment planning. However, it shows poorly on CSF and often does not provide a definitive diagnosis of CSF rhinorrhea. Computed tomography cisternography (CTC) is useful in identifying the site of leaks in multiple bone defects and single active leaks. MRH is useful in identifying intermittent leaks or meningoencephalocele.

CTC is a scan of the nasal cavity after intrathecal injection of iodinated contrast agent, where the patient is placed prone in the Trendelenburg position for approximately 5 minutes to achieve optimal cisterna filling (8). CTC is used to visualize lesions in the cisterna and intracerebroventricular space that are consistent with CSF density and is used to observe and assess CSF circulation condition. CTC is usually used for inactive nasal leaks or multiple bone defects, which should be compared with spiral CT performed before contrast injection (12). CTC is superior for demonstrating frontal or sphenoid sinus leaks due to the benefit of contrast agent accumulation in the sinus cavity; if the leak is located in the cribriform plate or olfactory groove, the contrast agent flows directly to the pharynx, making it difficult to detect the leak. Because of the thin bone in the ethmoid 
sinus, cribriform plate fracture fragments are not easily distinguished from the leaking contrast on the image, making image interpretation more difficult. Studies have reported that CTC has a broad sensitivity of $33 \%$ to $100 \%$ and a specificity of approximately $94 \%$ (13). The accuracy of CTC leak localization ranges between $85-92 \%$ for active leaks and $40 \%$ for inactive leaks (14). The main limitation of CTC is that only active CSF leakage can be found, while the discovery rate of intermittent or small leaks is low, and it requires rhinorrhea during examination. CTC requires contrast-containing CSF to pass through the leak in order to localize it, but the scan time is short and the bone tissue is well visualized. The low sensitivity is mainly attributed to the unfavorable visualization in the absence of an active leak, and other potential reasons are the high density of the bone adjacent to contrast and the high viscosity of the contrast agent that prevents leakage of contrast through the leak, allowing small leaks to be obscured (15). The disadvantages of CTC include the high radiation dose associated with multiple scans, the inherent risks of lumbar puncture, the potential adverse reactions of iodine contrast agent, and the risk of brain herniation and allergic reactions. CTC is highly specific and can directly show the morphology, size, location, and number of leak channels in CSF rhinorrhea. However, it does not provide a complete condition of the leak and does not display bony structures clearly, and therefore it should be combined with CT. For patients with acute meningitis or those with relative renal insufficiency, CTC can be combined with spiral CT to locate the leak.

MRH uses heavy T2-weighted sequences to study the relative high signal intensity of CSF in the arachnoid space by suppressing the background signal of soft tissue and brain parenchyma. It supports submillimeter wave acquisition and multiplanar reconstruction, which helps in the optimal assessment of skull base defects (16). Selecting the position for MRH when the CSF leak is more obvious, combined with the location of the fracture shown on $3 \mathrm{D}$ CT of the skull base, and observing the white CSF signal in the subarachnoid space helps to show the path of CSF leakage from the skull to the nasal cavity by showing a linear aqueous high-signal shadow connecting the cranial cavity to the leaky sinus. MRH can be considered positive when the CSF flow traces is between the subarachnoid space and the extracranial area with or without meningoencephalocele, with abnormal extension of the subarachnoid cisterns to the extracranial area (14). In contrast to T2WI alone, $\mathrm{MRH}$ imaging by $3 \mathrm{D}$ thin-layer imaging can show not only larger fistulas but also smaller fistulas not shown using CT and CSF rhinorrhea with inactive or multiple fistulas (17). For inactive CSF rhinorrhea, the location of the leak can be clearly shown and even smaller leaks can be accurately identified by examining changes in body position and increasing intracranial pressure by compressing the bilateral jugular veins, such that the CSF rhinorrhea becomes temporarily persistent. MRH is poor at showing bony structures and does not directly show bony defects, and therefore combining MRH with spiral CT has a complementary effect. A polypoid soft tissue shadow in the sinus cavity with an adjacent bone defect,which may be caused by nasal polyposis or nasal tumors, suggests a suspected meningoencephalocele and therefore a combination of history and multiple imaging methods is needed to clarify. Reddy confirmed that , CSF was discovered in the subarachnoid space connected to the extracranial space with or without intracranial meningeal herniation or brain parenchyma (11). Ecin's literature reports a diagnostic accuracy of $65 \%$, sensitivity of $56 \%$, and specificity of $77 \%$ for MRH (18). The positive rate of MRH in our group was $82.5 \%$ (33/40), but the location of the bone defect or fracture could not be determined. Combining MRH with spiral CT resulted in a $90 \%$ compliance rate for localization of the leak (19). In this study, combining MRH with spiral CT resulted in a positive rate of $82.5 \%(35 / 40)$ for the localization of the leak. The advantages of MRI is to improved soft tissue contrast, which helps to show herniated contents or meningoencephalocele through bone defects and assists with identification of sinus cavity CSF and inflammatory effusion, the former with lower T1WI signal.

CSF rhinorrhea relates to extracranial leaks that accompany bone and dural defects. Imaging studies is essential for localization of the leak site and establishment of preoperative planning. CSF rhinorrhea requires a systematic approach to evaluate skull base defects. Cranial spiral CT is the preferred imaging method, CTC for multiple bone defects, and MRH for intermittent leaks. The use of MRH combined with the CTC technique may improve the diagnostic rate in determining the location of CSF leaks and may also provide important clinical value in the surgical treatment planning of patients with CSF leaks. This study has the disadvantages of a small number of cases and a short follow-up period, and therefore further studies are needed to determine whether these imaging methods are worthy of further promotion. 


\section{Acknowledgments}

Funding: None.

\section{Footnote}

Reporting Checklist: The authors have completed the STROBE reporting checklist. Available at https://dx.doi. org/10.21037/apm-21-1994

Data Sharing Statement: Available at https://dx.doi. org/10.21037/apm-21-1994

Conflicts of Interest: All authors have completed the ICMJE uniform disclosure form (available at https://dx.doi. org/10.21037/apm-21-1994). The authors have no conflicts of interest to declare.

Ethical Statement: The authors are accountable for all aspects of the work in ensuring that questions related to the accuracy or integrity of any part of the work are appropriately investigated and resolved. The study was approved by the Ethics Committee of Clinical Investigation in the Tianjin Huanhu Hospital (No. 2021-049: the registration number of ethics board). All procedures performed in this study involving human participants were in accordance with the Declaration of Helsinki (as revised in 2013). Individual consent for this retrospective analysis was waived.

Open Access Statement: This is an Open Access article distributed in accordance with the Creative Commons Attribution-NonCommercial-NoDerivs 4.0 International License (CC BY-NC-ND 4.0), which permits the noncommercial replication and distribution of the article with the strict proviso that no changes or edits are made and the original work is properly cited (including links to both the formal publication through the relevant DOI and the license). See: https://creativecommons.org/licenses/by-nc-nd/4.0/.

\section{References}

1. Majhi S, Sharma A. Outcome of Endoscopic Cerebrospinal Fluid Rhinorrhoea Repair: An Institutional Study. Indian J Otolaryngol Head Neck Surg 2019;71:76-80.

2. Daudia A, Biswas D, Jones NS. Risk of meningitis with cerebrospinal fluid rhinorrhea. Ann Otol Rhinol Laryngol 2007;116:902-5.
3. Miner JR, Heegaard W, Mapes A, et al. Presentation, time to antibiotics, and mortality of patients with bacterial meningitis at an urban county medical center. J Emerg Med 2001;21:387-92.

4. Parmar H, Gujar S, Shah G, et al. Imaging of the anterior skull base. Neuroimaging Clin N Am 2009;19:427-39.

5. Alonso RC, de la Peña MJ, Caicoya AG, et al. Spontaneous skull base meningoencephaloceles and cerebrospinal fluid fistulas. Radiographics 2013;33:553-70.

6. Ommaya AK, Di Chiro G, Baldwin M, et al. Nontraumatic cerebrospinal fluid rhinorrhoea. J Neurol Neurosurg Psychiatry 1968;31:214-25.

7. Fang Z, Song M, Zhang Y. Endoscopic transnasal surgery for cerebrospinal fluid rhinorrhea: pilot study. Chinese Journal of Contemporary Neurology and Neurosurgery 2019;4:250-6.

8. Prosser JD, Vender JR, Solares CA. Traumatic cerebrospinal fluid leaks. Otolaryngol Clin North Am 2011;44:857-73, vii.

9. Schlosser RJ, Bolger WE. Nasal cerebrospinal fluid leaks: critical review and surgical considerations. Laryngoscope 2004;114:255-65.

10. Platt MP, Parnes SM. Management of unexpected cerebrospinal fluid leak during endoscopic sinus surgery. Curr Opin Otolaryngol Head Neck Surg 2009; 17:28-32.

11. Reddy M, Baugnon K. Imaging of Cerebrospinal Fluid Rhinorrhea and Otorrhea. Radiol Clin North Am 2017;55:167-87.

12. Lloyd KM, DelGaudio JM, Hudgins PA. Imaging of skull base cerebrospinal fluid leaks in adults. Radiology 2008;248:725-36.

13. Oakley GM, Alt JA, Schlosser RJ, et al. Diagnosis of cerebrospinal fluid rhinorrhea: an evidence-based review with recommendations. Int Forum Allergy Rhinol 2016;6:8-16.

14. Hiremath SB, Gautam AA, Sasindran V, et al. Cerebrospinal fluid rhinorrhea and otorrhea: A multimodality imaging approach. Diagn Interv Imaging 2019;100:3-15.

15. Goel G, Ravishankar S, Jayakumar PN, et al. Intrathecal gadolinium-enhanced magnetic resonance cisternography in cerebrospinal fluid rhinorrhea: road ahead? J Neurotrauma 2007;24:1570-5.

16. Hatipoğlu HG, Durakoğlugil T, Ciliz D, et al. Comparison of FSE T2W and 3D FIESTA sequences in the evaluation of posterior fossa cranial nerves with MR cisternography. Diagn Interv Radiol 2007;13:56-60.

17. Tuntiyatorn L, Laothammatas J. Evaluation of MR 
cisternography in diagnosis of cerebrospinal fluid fistula. J Med Assoc Thai 2004;87:1471-6.

18. Ecin G, Oner AY, Tokgoz N, et al. T2-weighted vs. intrathecal contrast-enhanced MR cisternography in the evaluation of CSF rhinorrhea. Acta Radiol 2013;54:698-701.

Cite this article as: Zhai X, Li L, Lu X, Li HY, Yang XL, Wang XD. Application of different imaging methods for the localization of cerebrospinal fluid rhinorrhea: a comparative study. Ann Palliat Med 2021;10(8):8797-8807. doi: 10.21037/ apm-21-1994
19. Yang B, Nie Y, Qin J. An analysis of diagnosis and treatment of cerebrospinal fluid rhinorrhea. Chinese Journal of Clinical Neurosurgery 2014;4:203-5.

(English Language Editor: B. Meiser) 